\title{
GMES - The EU Earth Observation Programme
}

\author{
Ondřej Mirovský \\ Czech Space Office, Prvního Pluku 17, Prague 8, 186 00, Czech Republic \\ mirovsky@czechspace.cz
}

\begin{abstract}
The paper describes evolution, recent development and structure of the Global Monitoring for Environment and Security (GMES) programme, which is a European tool how to bring data produced within Earth Observation capacities closer to daily use for numerous international, national and even regional users. GMES will help to ensure sustainable flow of accurate and timely data to monitor changes of our environment and will be a helpful tool to manage and coordinate fast emergency response.
\end{abstract}

Keywords: GMES, European Union, European Commission, European Space Agency, European Environmental Agency, services, data, environment, security.

\section{Introduction}

The planet Earth is recently going through ages of rapid change of its surface, biosphere, atmosphere and climate, which has impact on both nature and people inhabiting this planet. In order to be able to monitor these changes, Earth Observation (EO) gives us powerful tool how to get detailed information on global scale in a short of time.

European Union (EU) is in terms of environmental issues global leader and needs accurate and timely information to fulfil all monitoring and reporting demands as well as data for quick emergency response. Therefore, Global Monitoring for Environment and Security (GMES) as the European Initiative for the establishment of a European capacity for Earth Observation was launched.

\section{GMES - From the GMES Programme}

A key driving element, having contributed to the establishment of GMES, was the paradox of having so much data produced within current Earth Observation systems on one hand and lack of good quality and timely data delivered to decisions makers on the other hand. Thus, in 1998 in Baveno (Italy) representatives of numerous institutions in this field concluded together with European Commission (EC) and European Space Agency (ESA) to establish European capacity for Earth Observation named GMES.

However, it was not only need to ensure data for Europe, but GMES bears also greater geostrategic importance of having autonomous system not dependent on 
non-European systems, where still recently EU depends almost from $60 \%$ on foreign EO systems. EU commitment in this field is also a good tool to support European spaces industry, research and development while are all targeting to help to meet goals of the EU Lisbon strategy [1] and also EU 2020 strategy [2].

During last few years GMES has received wider importance within EU and in 2004 GMES was recognized in the Communication from the Commission to the European parliament and the Council (COM 2004 65) [3] followed by the resolution of the Parliament giving "green" light to further develop GMES.

Further on GMES found substantial basis to its development via finances from Seventh Framework Programme (FP7) in the domain of SPACE research. In the period 2007- 2013, 1.2 billion EUR were made available to develop GMES. In 2010 key step forward to make GMES a real programme was taken by the adoption of the regulation No. 911/2010 (Regulation of the European Parliament and of the Council on the European Earth monitoring programme (GMES) and its initial operations 2011-13), which entered into force on 9th November 2010 [4]. In accordance with the Regulation, the EU provides $€ 107$ million, of which $€ 43$ million are foreseen for the initial operation of GMES services and €64 million for the GMES Space Component [5].

\section{Architecture of GMES}

The GMES federates a wide range of observational networks and data providers, exploiting the most recent observation techniques and technologies, for developing edge-cutting information products to end-users. In principle, the GMES observational infrastructure composes of two main components - space and in situ.

\subsection{Space Infrastructure}

The space component shall ensure sustainable provision of satellite derived Earth observation data to all GMES services. The architecture of the component is derived from service requirements provided by the user communities. ESA and EUMETSAT are two main European actors in this area who should play the major role in coordination, implementation and operational running of the infrastructure [6].

Key elements of this component will be sets of 6 satellites systems named Sentinels, which shall cover all space born data needs for all services. These satellites will acquire radar and optical data, information on atmospheric chemistry and many other needs. First satellites on the orbit are expected in 2013. It is also a key aspect of benefits of GMES programme that Sentinel satellite systems are synergic logical follow up of some already existing satellite systems widely used in Europe (e.g. SPOT and ENVISAT).

\subsection{In-Situ Infrastructure}

The in situ component is based on an observation infrastructure owned and operated by the large number of stakeholders coordinated, in some cases, in the frame of European or international networks. In situ observation activities and associated infrastructure derive from a range of national, EU and international regulatory requirements and agreements or form part of research processes. None was created to meet the needs of 
GMES, and they cover a much wider field than the GMES services. By this reason European Environmental Agency was appointed to co-ordinate the consolidation of in-situ networks for GMES purposes [7].



Fig. 1. GMES Sentinel satellites [source ESA]

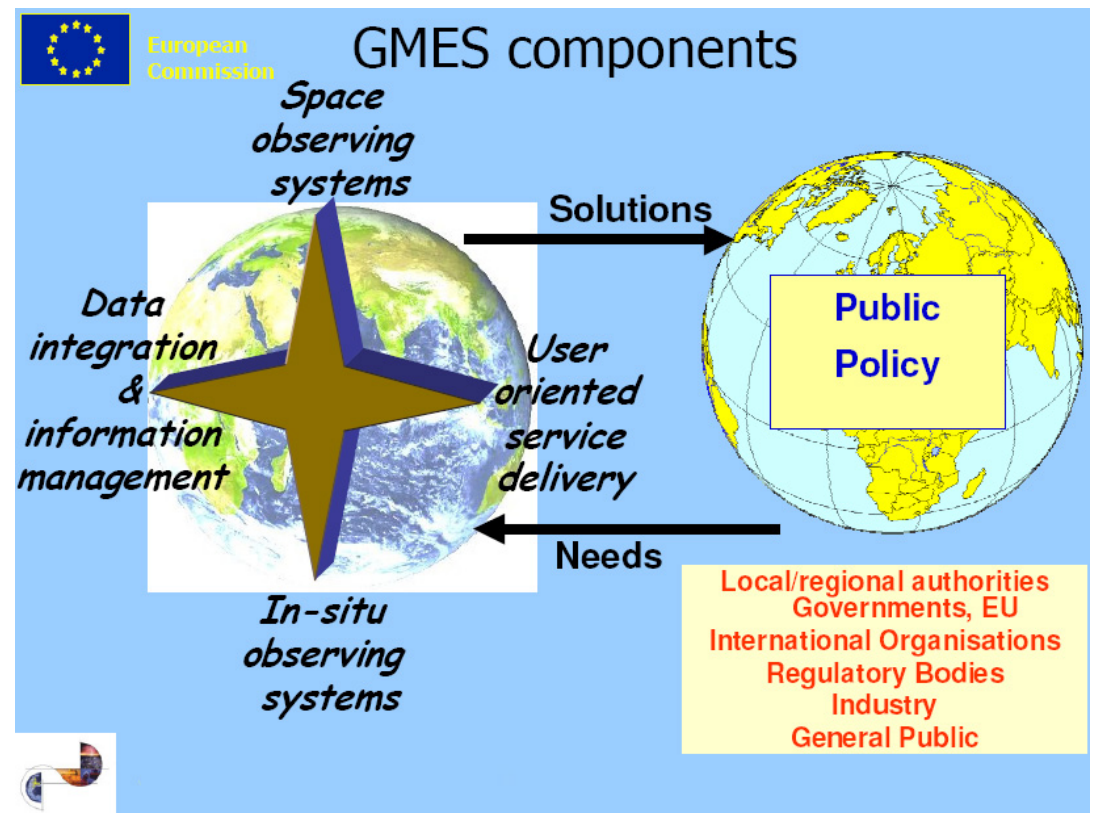

Fig. 2. GMES architecture [source EC] 


\subsection{Users}

Another key element of the GMES will be final users. Users are here to define their needs to both space and in-situ elements in order to get from the system such data they can instantly use for their daily needs. User-driven principle shall be applied in both elements - for the design of satellite systems as well as on in-situ data processing to final users. What European users need most are ready-made tailored data.

\section{GMES Services}

Bringing GMES into reality of daily life involves sets of services, which are now in pilot phase with the future transition into operational services. Today we have six basic GMES services under development tackling most needed information for European users.

Land monitoring service is now being developed under GEOLAND 2 [7] project and it is dedicated to cover land monitoring needs for Europe including topics as land cover changes, agro-environmental issues, spatial planning, forest monitoring etc. For the domain of marine applications project MyOcean is now processed to cover monitoring of the ocean and seas in order to get better data on maritime security, natural recourses, oil spill prevention etc [8].

Emergency response service of GMES is covered by project SAFER [9], which gathers activities towards to a rapid mapping and provision of online information during emergency situations. The scope of this service goes even on global level, when this service has the potential to work worldwide. Atmosphere services of GMES are recently under MACC [10] project, when core of this task is to deliver data on air quality, climate, monitor sand and dust storms, UV radiation risks etc. Lastly, G-MOSAIC project is now running to cover security GMES services. Core of activities is in the provision of geo-spatial information in support of EU external relation policies for Security related activities [11].

Recently, another service covering GMES climate change monitoring is prepared to be initiated in 2012 .

\section{GMES - Operational EO Service}

After twelve years GMES has now entered its operational phase through many research projects financed by the EU, ESA and Member States aimed to develop future operational services and infrastructure. The services are being developed to meet the needs of a wide range of users who rely on accurate environmental and security data and information. Operational, continuous and sustainable delivery of information has not yet been achieved. Further investment is therefore necessary, in Space infrastructure in particular, in order to fill the remaining gaps in GMES services and to guarantee their long-term sustainability and reliability. In addition, a common approach between the various partners involved in the development of GMES needs to be further enhanced, to avoid the possibility of a duplication of efforts. GMES is also creating opportunities for increased private sector usage of new information sources. It will trigger partnerships between research and service providers, many of them small and 
medium enterprises. Thus, while not likely in the short to medium term, the development of market opportunities could eventually determine the proportion of public investment [12].

\section{Conclusion}

Earth Observation encompasses a powerful set of advanced technologies which in combination with in situ (ground-based, airborne etc.) measurements provides products and services supporting solutions to international challenges such as security threats, environmental degradation and climate change. The GMES initiative reflects the European decision to develop its own, independent observation capabilities. At this time GMES starts the transition into real operational services delivering data where needed and has a unique potential to be a very successful approach how to maintain our planet safe and healthy. For its success, future strong commitment of its main key players - European Commission and European Space Agency is needed together with the voice of member states of the EU and other international bodies.

\section{References}

1. EU Lisbon strategy, http://ec.europa.eu/archives/growthandjobs_2009/

2. EU 2020 strategy, http: / / ec . europa. eu / europe2020/index_en.htm

3. COM (2004) 65, COMMUNICATION FROM THE COMMISSION TO THE EUROPEAN PARLIAMENT AND THE COUNCIL, Global Monitoring for Environment and Security (GMES): Establishing a GMES capacity by 2008 - (Action Plan (2004-2008))

4. Regulation of the European Parliament and of the Council on the European Earth monitoring programme (GMES) and its initial operations 2011-13,

http: / / download.esa.int/docs / GMES / GMES_Regulation_911-2010_FINAL.pdf

5. ESA GMES, http: //www. esa.int/esaLP/SEMEAN1PLFG_LPgmes_0.html

6. GMES Observational Infrastructure, http://ec.europa.eu/gmes/obser_infra.htm

7. Geoland 2, http://www.gmes-geoland. info/

8. MyOcean, http: / /www. myocean.eu.org/index.php/project/objectives

9. SAFER, http://safer. emergencyresponse.eu/site/FO/ scripts/myFO_accueil.php?lang=EN

10. MACC, http://www.gmes-atmosphere.eu/

11. G-MOSAIC, http://www.gmes-gmosaic.eu/

12. Kolar, J., Mirovsky, O.: The Czech EU presidency: a gateway to GMES for users from Central and Eastern Europe, Window on GMES (March 2009) ISSN 2030-5410, http: / / www . boss4gmes . eu / index .php?id=103\&no_cache=1 